\title{
UNCONVENTIONAL TAIL CONFIGURATIONS FOR TRANSPORT AIRCRAFT
}

\section{A. Sánchez-Carmona, C. Cuerno-Rejado, and L. García-Hernández}

\author{
Escuela Técnica Superior de Ingenieros Aeronáuticos \\ Department of Aircraft and Space Vehicles \\ Universidad Politécnica de Madrid \\ 3 Plaza Cardenal Cisneros, Madrid 28040, Spain
}

\begin{abstract}
This article presents the bases of a methodology in order to size unconventional tail configurations for transport aircraft. The case study of this paper is a V-tail configuration. Firstly, an aerodynamic study is developed for determining stability derivatives and aerodynamic forces. The objective is to size a tail such as it develops at least the same static stability derivatives than a conventional reference aircraft. The optimum is obtained minimizing its weight. The weight is estimated through two methods: adapted Farrar's method and a statistical method. The solution reached is heavier than the reference, but it reduces the wetted area.
\end{abstract}

\section{NOMENCLATURE}

$\begin{array}{ll}\mathrm{AR}_{\mathrm{HTP}} & \text { Aspect ratio of horizontal projection of the tail } \\ \mathrm{AR}_{\mathrm{VTP}} & \text { Aspect ratio of vertical projection of the tail } \\ b & \text { Span measured along the surface } \\ b_{\text {ref }} & \text { Reference span } \\ b_{\mathrm{st}} & \text { Real span: } b / \cos \Lambda_{0.25} \\ b_{t} & \text { Torsion box width } \\ c_{\text {contr }} / c & \text { Relative chord of tail controls } \\ C_{\mathrm{fle}} & \text { Fixed leading edge coefficient for composite weight reduction } \\ C_{\mathrm{fte}} & \text { Fixed trailing edge coefficient for composite weight reduction } \\ C_{L \alpha} & \text { Lift curve slope } \\ C_{m \alpha} & \text { Longitudinal static stability derivative } \\ C_{n \beta} & \text { Lateral static stability derivative } \\ C_{r} & \text { Root chord }\end{array}$

(C) The authors, published by EDP Sciences. This is an Open Access article distributed under the terms of the Creative Commons Attribution License 4.0 (http://creativecommons.org/licenses/by/4.0/). 
$C_{\text {tef }} \quad$ Trailing edge flap coefficient for composite weight reduction

$C_{Y \beta} \quad$ Lateral force coefficient derivative with respect sideslip angle

$c(y) \quad$ Chords distribution along $y$ axis

$F_{\text {TU }} \quad$ Ultimate tensile of material

$F_{z} \quad$ Shear force

$g \quad$ Gravity

$h_{t} \quad$ Torsion box height

$K \quad$ Factor of tail weight distribution

$K_{\rho H} \quad$ Horizontal tail density factor

$K_{\rho V} \quad$ Vertical tail density factor

$k \quad$ Diagonal tension factor

$k_{\mathrm{fle}} \quad$ Fixed leading edge factor

$k_{\text {rib }} \quad$ Rib weight factor

$k_{\text {slot }} \quad$ Number-of-flap-slot factor

$k_{\text {sup }} \quad$ Kind-of-flap factor

$M_{x} \quad$ Bending moment

MAC Mean aerodynamic chord

MTOW Maximum takeoff weight

$N \quad$ Compression force per meter

$n_{\text {ult }} \quad$ Ultimate load factor

$q \quad$ Shear flow

$q_{D} \quad$ Dynamical pressure for divergence speed

$q_{\text {lim }} \quad$ Limit shear flow

$q_{\text {ref }} \quad$ Reference dynamic pressure

$S_{\text {fle }} \quad$ Fixed leading edge area

$S_{\text {fte }} \quad$ Fixed trailing edge area

$S_{\text {HTP }} \quad$ Area of horizontal projection of the tail

$S_{\text {tail }} \quad$ Tail area

$S_{\text {tef }} \quad$ Trailing edge flap area

$S_{\mathrm{VTP}} \quad$ Area of vertical projection of the tail

$T \quad$ Torsor moment

$t / c \quad$ Relative thickness

$t_{L} \quad$ Spar thickness

$t_{\text {ref }} \quad$ Reference thickness

$t_{\text {root }} \quad$ Root chord thickness

$t_{\text {tip }} \quad$ Tip chord thickness

$V_{\mathrm{eH}} \quad$ Volume coefficient of horizontal projection of the tail

$V_{\mathrm{eV}} \quad$ Volume coefficient of vertical projection of the tail

$W_{\text {attach }}$ Attachments weight

$W_{\text {box }} \quad$ Torsion box weight

$W_{\text {HTP }} \quad$ Weight of horizontal projection of the tail

$W_{\text {joints }} \quad$ Joints weight

$W_{\text {ref }} \quad$ Reference weight 


$\begin{array}{ll}W_{\text {rib }} & \text { Ribs weight } \\ W_{\text {tail }} & \text { Tail weight } \\ W_{\text {VTP }} & \text { Weight of vertical projection of the tail } \\ w(y) & \text { Tail weight distribution } \\ \Gamma & \text { Dihedral angle } \\ \delta_{\text {id }} & \text { Joints and fasteners weight factor } \\ \Lambda_{0.25} & 1 / 4 \text { chords line sweep } \\ \Lambda_{0.25 H} & 1 / 4 \text { chords line sweep of horizontal projection } \\ \Lambda_{\text {le }} & \text { Leading edge sweep } \\ \lambda & \text { Taper ratio } \\ \rho & \text { Tail structure density } \\ \Omega_{\mathrm{fle}} & \text { Specific weight of fixed leading edge } \\ \Omega_{\mathrm{fte}} & \text { Specific weight of fixed trailing edge } \\ \Omega_{\mathrm{ref}} & \text { Reference specific weight } \\ \Omega_{\mathrm{tef}} & \text { Specific weight of trailing edge flaps }\end{array}$

\section{INTRODUCTION}

The air transport over Europe is expected to grow on around $4 \%$ annually during the next 20 years. This increasing of passengers will be higher on the AsiaPacific zone, which will be around $6 \%$ annually $[1,2]$. So, it is predictable that the aircraft demand will increase too. In fact, the number of aircraft in service on 2032 will be around 38,000 [1], which is approximately double of the current value. In addition, the flight segment more demanded will be single aisle, for instance, A320 or B737, with around 22,000 new deliveries [1].

On the other hand, the environmental impact of aviation lies on two main aspects: noise and emission of gases and particulates to the atmosphere which contributes to climate change [3]. Taking into account this fact and the air transport traffic forecast, the European Union is taking action to reduce aviation emissions in Europe and working with the international community to develop measures with global reach. One result of this worry is the resolution of the ICAO (International Civil Aviation Organization) Assembly on October of 2013, which promotes and facilitates the development and sustainable fuel for aviation [4].

Another possibility to reduce the emissions of the aircraft is reducing the fuel consumption. The emissions are proportional to fuel consumption. This supposes that aircraft in the future must be more efficiently designed and more environment-friendly. By the way, reductions on fuel consumption suppose savings on operating costs. Until now, some different strategies have been followed for carrying out this objective [5]. The engines, turbofan, and turboprop have been developed for reducing the fuel consumption itself. The incorporation of composite materials into the aircraft manufacturing reduces the structural weight and, consequently, the consumption and costs of the operation. Furthermore, the 
optimization of the trajectories followed by the aircraft allows improving the efficiency of the travel, for instance, the SESAR (Single European Sky ATM (Air Traffic Management) Research) programme works on it [6]. However, the aircraft configuration hardly has changed along the history, which consists on a slender fuselage, a high aspect ratio wing, and stabilizing surfaces attached to the tail cone [7]. It is true that the position of the engines has varied depending on the design. But only two possibilities have been considered: podded-engines under the wings or attached to the rear fuselage.

It seems that the strategies presented are reaching a limit [8]. New strategies, such as biofuels or innovative technologies, are needed to reduce the environment impact of aviation. Innovative aircraft configurations are one of these new strategies. Probably, for the near future, drastic changes in configurations are not possible; so, unconventional tail configurations could be a nice solution. The configurations installed along the history are considered planar, which are conventional tail, T-tail, and cruciform tail. These classical empennage designs have not reached an absolute optimum, because the investigations were made from a specific point of view (weight, aerodynamics, configuration, etc.) [9]. Nonplanar tail concepts encompass a variety of configurations including biplanes, box-planes, ring-tails, joined wings, and horizontal tail with winglets. These configurations need to be studied for determining their potential benefits $[10$, 11]. The initial case study will be centered on a V-tail configuration, which is the simplest unconventional configuration. Therefore, the work consists in designing a $\mathrm{V}$-tail which develops equivalent moments than the reference aircraft conventional tail surfaces in order to analyze advantages and drawbacks.

\section{REFERENCE AIRCRAFT}

The reference aircraft is going to be used for two objectives: validating the procedures which are going to be employed for the V-tail and measuring its lateral and longitudinal static stability for guaranteeing, at least, the same stability behavior to the V-tail. The reference aircraft has been selected according to the air traffic forecast presented at the beginning. It says that single aisle aircraft will be the most demanded. Thus, it seems justified to choose an aircraft similar to A320 or B737. In this context, the Institute of Aerospace Systems (ILR) of RWTH Aachen University is developing a project titled: Central Reference Aircraft System for Research Community. The goal of this project is building a Central Reference Aircraft data System available to the larger aircraft design research community. This idea appears because the industry has a restricted data authorization and this supposes that academia has limited availability of consistent reference aircraft data in research projects. Nowadays, this project presents a unique aircraft called CSR-01, which has similar performances and 
size than the actual single aisle aircraft. Hence, this aircraft will be taken as reference for this study. All the data necessary of CSR-01 for this study are known and included in the corresponding reference [12].

\section{METHODOLOGY}

The design procedure followed in this study consists of solving the aerodynamic problem first. Then, the aerodynamic forces carry the loads to the tail structure. The tail structure must support these aerodynamic forces and, consequently, it must have a determined weight. The idea is to find a geometry with minimum weight which achieves the stability constraints imposed by the reference aircraft. These schema could be applied to any unconventional configuration; but this paper focuses on V-tail. The aircraft is going to be modeled as a wing and a tail. This decision is based on the fact that the reference wing always is the main wing; and also, some relevant parameters, as volume coefficient, need the existence of the main wing in order to be possible to calculate them. Because of all these aspects, the tails are not analyzed in an isolated way. Figure 1 shows the geometry of the main wing of the reference aircraft and the model used.

The work may consist of studying all the critical load cases in conventional tails, both HTP (Horizontal Tail Plane) and VTP (Vertical Tail Plane), which will be simulated through an aerodynamic software. These load cases can be summed up as follows [14]:

- for VTP, critical engine failure, Dutch roll, lateral gusts, yawing manoeuver and minimum control speed at ground; and

- for HTP, longitudinal gusts, maximum control deflection, and pitching manoeuver.

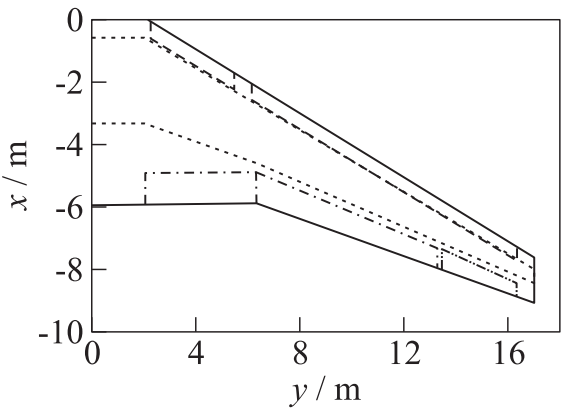

(a)

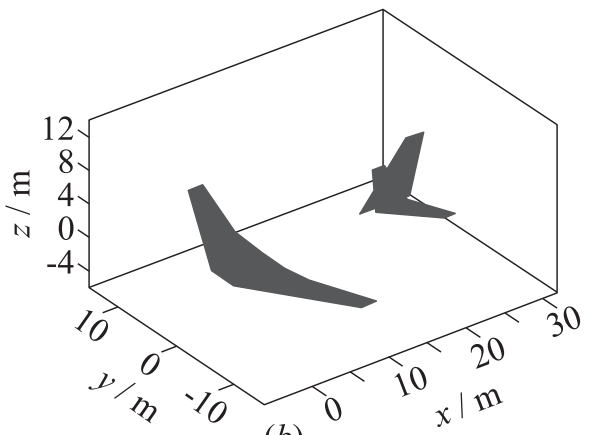

(b)

Figure 1 Reference aircraft CSR-01: (a) wing planform; and (b) 3D model for Tornado [13] 
If it is possible to analyze all these load cases, it will be sure that the critical case will be taken into account. The critical case can vary depending on the tail geometry. The procedure should analyze all the cases and take the most restrictive. This paper just develops one load case: maximum control deflection at $V_{A}$ (design maneuvering speed, which is the stall speed multiplied by the square root of the limit and positive load factor). Probably, this will not be the critical case, but the methodology that should be followed for the other cases would be analogous.

Some hypotheses have been made for facilitating the study. In spite of that, these hypothesis can be taken as new freedom degrees for future works. The first constraint is not to vary the center of gravity. Clearly, the center of gravity should change depending on the tail weight, but this fact would complicate too much the procedure. A similar reasoning is applicable to aerodynamic center. Its location is influenced by the tail geometry. Again, it has been decided not to take into account this effect for easier comprehension of the results. The last hypothesis is that the tail apex point will be at the same distance from the main wing as the tail of the reference aircraft.

\subsection{Geometry-Design Parameters}

First of all, it is necessary to establish the geometrical parameters which are considered in this study. These are root chord, semispan, taper ratio, and dihedral angle. The first three parameters allow modifying the tail planform and dihedral angle is the main parameter of a V-tail which makes this tail unconventional. Another parameter that could have been chosen is sweep angle. But it was decided to maintain the reference value because a previous analysis showed that it has hardly influenced the results.

An important parameter that varies implicitly with the tail planform is the moment arm. This parameter is defined as the distance between the point $1 / 4$ of the mean aerodynamic chord of the tail and the same point of the main wing. The mean aerodynamic chord and its position depend on the planform geometry. However, the high dihedral angles of a V-tail modifies slightly the classical expressions for mean aerodynamic chord and its position, specifically the position along the $y$ axis. Usually, it is referred to an axis along the surface; but the aircraft $y$ axis is perpendicular to the symmetry plane. Hence, the $y$ positon of the mean aerodynamic chord should be multiplied by a cosine of dihedral angle. Having in mind these explanations, the effect of the geometry on the tail moment arm is clearer. It is necessary to point out some explanations about the definitions of the four design parameters selected. All usual definitions are valid for conventional geometries, which include small dihedral angles. For instance, the usual definition of span is taken on the planform. In this study, span is defined along the surface, notwithstanding that each side of the surface points 
in different directions. The other three parameters (root chord, taper ratio, and dihedral angle) are taken as the usual definitions because this unconventional geometry does not affect them.

\subsection{Aerodynamics}

The aerodynamic problem has been solved with Tornado software [13]. Tornado is a Vortex Lattice Method for linear aerodynamic wing design applications in conceptual aircraft design or in aeronautical education. By modeling all lifting surfaces as thin plates, Tornado can be used for most aerodynamic derivatives for a wide range of aircraft geometries. Tornado allows to simulate multiple wing configuration, which is necessary for this study. This software has been used to obtain $C_{m \alpha}$ and $C_{n \beta}$. In addition, Tornado gives aerodynamic forces on each panel and from here, shear force $\left(F_{z}\right)$, bending moment $\left(M_{x}\right)$, and torsion moment $(T)$ distributions have been calculated. Note that torsor moments have been determined with respect to the half-chord line. Force and moment distribu-
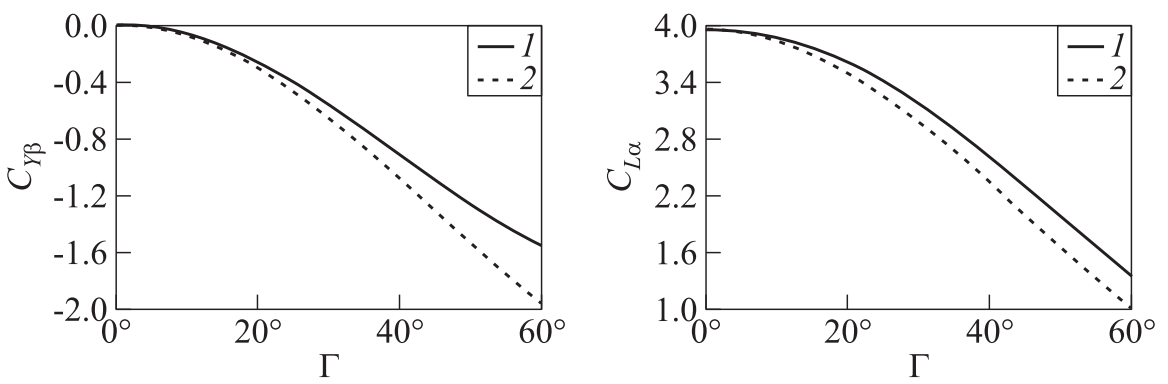

(a)
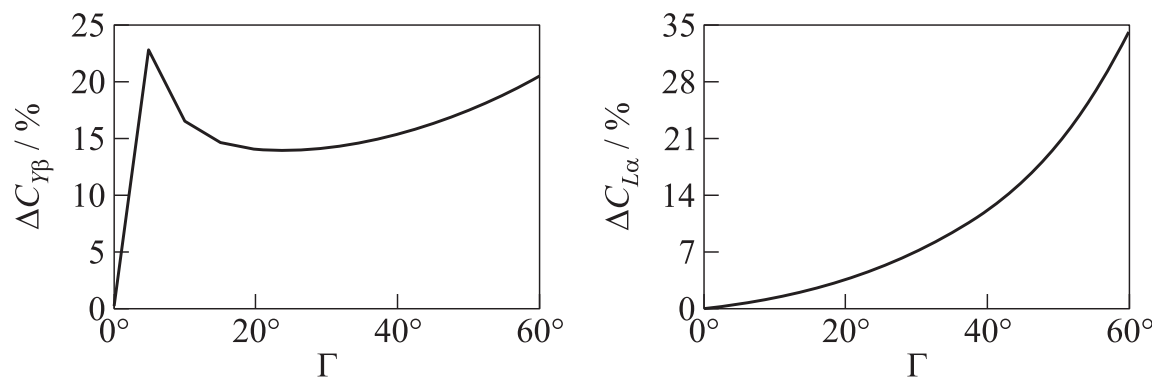

(b)

Figure 2 Stability derivatives comparison between Tornado (1) and NACA (National Advisory Committee for Aeronautics) theory (2) 
tions are calculated taking into account the force generated along the surface's normal. In principle, Tornado is valid for incompressible flow and small angle of attack. The Prandtl-Glauert correction has been used to improve the results in the high subsonic regime.

In order to validate somehow the results obtained with Tornado, reference [15] shows an analytical method of determining longitudinal and lateral behavior of a V-tail. More precisely, $C_{L \alpha}$ and $C_{Y \beta}$ are the derivatives which are going to be compared. The accuracy of this method is lower for high dihedral angles, but it describes the tendency of the derivatives. It is possible to see that Tornado follows the same behavior (Fig. 2). In spite of that, the results of stability derivatives are going to be referred to the reference tail results. In this way, the study is developed in qualitatively form for avoiding inaccuracies of the software for high angles presented in Fig. $2 b$.

\subsection{Weight}

The weight is the goal function of this procedure. The idea is to find a combination of dihedral angle, span, root chord, and taper ratio for which the weight will be minimum, assuring that this final combination will have at least the same static stability properties as the reference aircraft with conventional tail.

The method used in this paper for estimating the weight of each configuration is a variation of the method presented in [16]. This method was developed by Egbert Torenbeek for wings, instead of tails. It is based on sizing the torsion box of the wing in such a way that it resists the aerodynamic forces at limit load factor. The aerodynamic forces considered for this method are the bending moment and shear force caused by the lift distribution along the wing. Because these forces are maximum at the root chord, the method sizes all the wing supposing that the stresses are constant along the span and equal to the stress at a root. In addition, Torenbeek adds several terms based on statistics for determining the weights corresponding to surface controls, attachments, aeroelastic phenomena, etc. Finally, an increment of weight is included associated to fail safe and safe life requirements. This method has been applied to the wing of the reference aircraft for calibration. Note that current wings present a high percentage of composite materials; so, different reduction factors are needed to be applied to the weight depending on the component of the wing. The result is presented in Table 1 and one may see that the estimated weight slightly overestimates the real weight.

In spite of the good results of this method for the wing, it is not possible to use it directly for estimating the tailplane weights. But this case study of the main wing is used to calibrate some parameters which are going to be used for estimating the tail surface weight. The suggested modified method sizes the torsion box taking into account bending moments, shear forces, and torsor 
Table 1 Wing estimated weight through Torenbeek method for reference aircraft

\begin{tabular}{ccc}
\hline Estimating weight, $\mathrm{kg}$ & Real weight, $\mathrm{kg}$ & $\Delta$ \\
\hline 8411 & 8097 & $+3.88 \%$ \\
\hline
\end{tabular}

moments. Bending moment generates a distribution of axial force per meter expressed in

$$
N=\frac{M_{x}}{h_{t} b_{t}}
$$

where $h_{t}$ and $b_{t}$ are the height and the length of the torsion box. It is supposed that the torsion box is approximately rectangular. These measurements vary along the span according to the corresponding chord. In addition, it is supposed the typical positions of front and rear spars, which are $11 \%$ and $55 \%$ of chord length, respectively. Furthermore, torsor moment and shear force generate a distribution of shear flow along the panels and the spars of the torsion box. It is supposed that the highest shear flow is reached in the front spar and this maximum value is constant along the front spar itself and, also, that spar caps area are much smaller than extrados' area:

$$
|q|=\left|\frac{T}{2 h_{t} b_{t}}\right|+\left|\frac{F_{z}}{2 h_{t}}\right| .
$$

So, the two spars are going to be sized according this load.

Regarding extrados and intrados, it is supposed that the two panels present the same geometry and are sized according to the compression loads. This geometry is composed by two spar caps, which represent front and rear spar, and stringers with $Z$ section. Over these stringers, there is a sheet which forms extrados and intrados, respectively. Focusing on the spars, they are formed by two spar caps, which coincide with the extreme spar caps of extrados and intrados, and a sheet that connect them. In addition, this sheet is reinforced through stiffeners, which are located every $0.168 \mathrm{~m}$ along front and rear spars, respectively. This is a typical value for commercial aircraft. The spars are sized according to Kuhn et al. hypothesis [17], which says that shear stress in a sheet can be divided into pure diagonal tension and pure shearing. The factor $k$ represents the percentage of diagonal tension which the sheet is subjected (Fig. 3).

Furthermore, following Von Mises criterion, admissible stress can be expressed through

$$
\tau_{\text {admissible }}=\frac{F_{\mathrm{TU}}}{\sqrt{3+k^{2}}}
$$



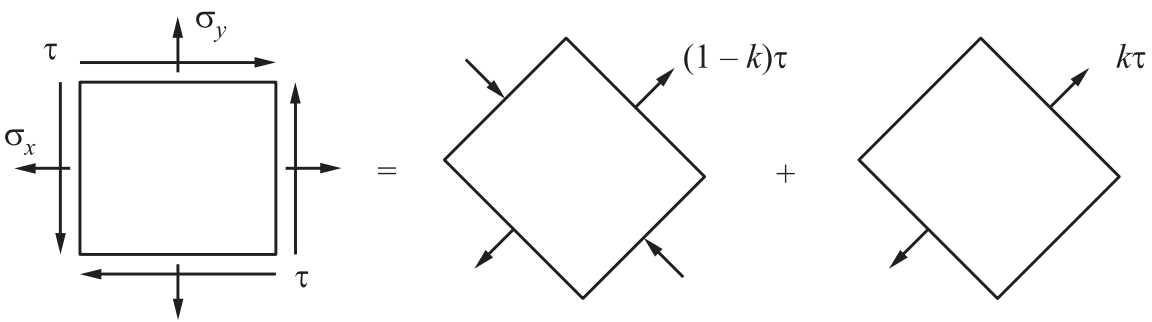

Figure 3 Shear stress divided into pure shear and pure diagonal tensions

as a function of factor $k$ and ultimate stress for the corresponding material, which is an aluminium alloy. On the other hand, the admissible stress should be equal to the ultimate load with a safety factor of $5 \%$. The ultimate load is the limit load multiplied by 1.5. The limit load should be the most critical of all the load cases simulated (expression (1)), but because this study contemplates only one load case, the stresses developed by this load case are taken as the limit ones. The following expression shows this way of obtaining the admissible stress:

$$
\tau_{\text {admissible }}=1.05 \cdot 1.5 \frac{q_{\text {lim }}}{t_{L}} .
$$

Thus, it is possible to size the spars connecting these last expressions with Kuhn et al. hypothesis and, consequently, determining the weight of the spars.

As it has been explained before, the extrados and the intrados are identical; so, this study sizes the extrados and immediately, the intrados is the same. The sizing criterion is the optimal Farrar factor [18] which minimizes the structural weight. This criterion supposes that both global and local bending failures are reached simultaneously. The corresponding Farrar factor which imposes this fact is 0.95. Again, considering that the unique load case simulated is the critical one, it is possible to size each section of the wing according to the corresponding ultimate load which is subjected. So, the weight of each section is the addition of the extrados, intrados, and front and rear spar weights.

Once the sizing process has been finished, the total weight of the torsion box is going to be calculated. It has been supposed that the structure has been made of composite material but maintaining the same geometry as it has been reached with this procedure, determining in this way the term $W_{\text {box }}$ which is going to be used later. This weight has to be added to the other terms of Torenbeek's method which are applicable to this type of tail. These terms are: fixed leading edge, fixed trailing edge, and trailing edge flap. The elevator is considered as a trailing edge flap from the standpoint of Torenbeek's method. These terms are calculated through a multiplication of a constant by their planform area. The values of all the constants are indicated in Table 2, and have been taken according to $[16]$ : 
Table 2 Values of the parameters used to calculate fixed leading edge, fixed trailing edge, and elevator weights

\begin{tabular}{|c|c|c|c|c|c|c|c|c|c|}
\hline$k_{\mathrm{fle}}$ & $\begin{array}{r}\Omega_{\mathrm{ref}} \\
\mathrm{N} / \mathrm{m}^{2} \\
\end{array}$ & $\begin{array}{c}q_{D} \\
\mathrm{~N} / \mathrm{m}^{2} \\
\end{array}$ & $\begin{array}{l}q_{\mathrm{ref}} \\
\mathrm{N} / \mathrm{m}^{2}\end{array}$ & $b_{\mathrm{st}}, \mathrm{m}$ & $\begin{array}{l}\text { MTOW, } \\
\mathrm{N}\end{array}$ & $\begin{array}{c}W_{\text {ref }}, \\
\mathrm{N}\end{array}$ & $\begin{array}{c}b_{\text {ref }}, \\
\mathrm{m}\end{array}$ & $k_{\text {sup }}$ & $k_{\text {slot }}$ \\
\hline 1 & 56 & 13218 & 30000 & $\begin{array}{c}b / \cos \Lambda_{0.25} \mathrm{~m} \\
\text { (it depends on the span) }\end{array}$ & 755370 & $10^{6}$ & 50 & 1 & 1 \\
\hline
\end{tabular}

Table 3 Values of the parameters used to calculate ribs, attachments, and joints weights

\begin{tabular}{ccccccccc}
\hline$k_{\text {rib }}$ & $\begin{array}{c}S_{\text {tail }}, \\
\mathrm{m}^{2}\end{array}$ & $\begin{array}{c}t_{\text {ref }}, \\
\mathrm{m}\end{array}$ & $\begin{array}{c}t_{\text {root }}, \\
\mathrm{m}\end{array}$ & $\begin{array}{c}t_{\text {tip }}, \\
\mathrm{m}\end{array}$ & $\begin{array}{c}\rho_{\text {mat }}, \\
\mathrm{kg} / \mathrm{m}^{3}\end{array}$ & $\begin{array}{c}g, \\
\mathrm{~m} / \mathrm{s}\end{array}$ & $n_{\text {ult }}$ & $\delta_{\text {id }}$ \\
\hline 0.003 & $\frac{1+\lambda}{2} C_{\text {root }} b$ & 0.45 & $0.12 C_{\text {root }}$ & $0.12 C_{\text {tip }}$ & 1900 & 9.81 & 3.75 & $10^{-3}$ \\
\hline
\end{tabular}

$$
\begin{aligned}
& \Omega_{\mathrm{fle}}=3.15 k_{\mathrm{fle}} \Omega_{\mathrm{ref}}\left(\frac{q_{D}}{q_{\mathrm{ref}}}\right)^{0.25}\left(\frac{b_{\mathrm{st}} \mathrm{MTOW}}{W_{\mathrm{ref}} b_{\mathrm{ref}}}\right)^{0.145} ; \\
& \Omega_{\mathrm{fte}}=2.6\left(\Omega_{\mathrm{ref}}+40\right)\left(\frac{\mathrm{MTOW} b_{\mathrm{st}}}{W_{\mathrm{ref}} b_{\mathrm{ref}}}\right)^{0.0544} ; \\
& \Omega_{\mathrm{tef}}=1.7 k_{\mathrm{sup}} k_{\mathrm{slot}} \Omega_{\mathrm{ref}}\left(1+\left(\frac{\mathrm{MTOW}}{W_{\mathrm{ref}}}\right)^{0.35}\right) .
\end{aligned}
$$

Furthermore, the ribs weight has been taken into account also and additional terms like attachments and joints have been considered (Table 3). Again, these weights have been calculated according to [16]. The expressions and parameters used are specified as follows:

$$
\begin{aligned}
W_{\text {ribs }} & =\rho_{\text {mat }} g k_{\text {rib }} S_{\text {tail }}\left(t_{\text {ref }}+\frac{t_{\text {root }}+t_{\text {tip }}}{2}\right) ; \\
W_{\text {attach }} & =0.0003 n_{\text {ult }} \mathrm{MTOW} \frac{S_{\text {tail }}}{S_{\text {refw }}} ; \\
W_{\text {joints }} & =1.20 \rho g S_{\text {tail }} \delta_{\text {id }} .
\end{aligned}
$$

To sum up, the final expression of tail weight estimation is the following:

$$
\begin{aligned}
W_{\text {tail }}=W_{\text {box }}+W_{\text {ribs }}+1.10\left(\Omega_{\mathrm{fle}} S_{\mathrm{fle}} C_{\mathrm{fle}}+\Omega_{\mathrm{fte}} S_{\mathrm{fte}} C_{\mathrm{fte}}\right. \\
\left.+\Omega_{\mathrm{tef}} S_{\mathrm{tef}} C_{\mathrm{tef}}\right)+W_{\text {attach }}+W_{\text {joints }} .
\end{aligned}
$$

The parameters which appear in Eq. (2) are specified in Table 4 . The parameters $S_{i}$ are the area of the element measured along the surface and the 
Table 4 Values of the parameters used to calculate the final weight tail

\begin{tabular}{cccccc}
\hline$S_{\text {fle }}, \mathrm{m}^{2}$ & $S_{\text {fte }}, \mathrm{m}^{2}$ & $S_{\text {tef }}, \mathrm{m}^{2}$ & $C_{\text {fle }}$ & $C_{\text {fte }}$ & $C_{\text {tef }}$ \\
\hline $0.04 S_{\text {tail }}$ & $0.036 S_{\text {tail }}$ & $0.25 S_{\text {tail }}$ & 0.80 & 0.80 & 0.85 \\
\hline
\end{tabular}

parameters $C_{i}$ are the factors included in order to consider weight reduction caused by composite materials, as it has been commented previously. Note that the third term of the equation is multiplied by 1.10. This is a correction factor recommended in [16] in order to take into account miscellaneous items. But it should be calibrated if more information were available.

It is necessary to indicate that the forces of each section have two sources: aerodynamics and structural weight. The aerodynamic effects are described in the following section. Because of the fact of taking into account the structural weight, inertial effects transmit loads to the structure. It is supposed a weight distribution according to

$$
w(y)=K c(y)^{1.2} .
$$

The constant is adjusted for guaranteeing that the whole weight of the tail coincides with the estimated one. So, integrating Eq. (3) along the span and equalling to total weight, the constant is determined. Considering this weight distribution, the forces per meter caused by it are calculated. As it is possible to deduce from this explanation, determining the weight of a configuration is an iterative procedure. When the initial weight is equal to the final estimated weight $\left(W_{\text {tail }}\right)$, taking into account a tolerance, it says that the procedure converges.

In order to validate the results obtained by the previous method, another procedure is going to be presented. The main drawback of the previous method is that it does not take into account lateral forces for determining the weight, since just one symmetrical load case has been simulated. So, if this load case is not the critical one, the geometry reached through this method and its weight are not correct. Because of that, a rapid method for estimating the weight of a V-tail has been developed to learn how far this load condition is from the critical one. This method is based on statistical formulas applied to horizontal and vertical tails [19]. The equations presented in this section are developed based on four sources: direct relationship between weight of a component and its average density, actual published data on weight of various components, derived empirical factors, and published empirical equations. The first and second sources make the technique very accurate and reliable. However, the third source indicates that there must be a calibration of the results to adjust the empirical factors.

The weight of the horizontal tail is a function of horizontal tail planform area, tail maximum thickness-to-chord ratio, tail configuration, construction material, elevator chord, and other horizontal tail geometries such as aspect ratio, taper ratio, and sweep angle. Its weight is calculated as follows (in kilograms): 


$$
\begin{aligned}
& W_{\mathrm{HTP}} \\
& =S_{\mathrm{HTP}} \mathrm{MAC}\left(\frac{t}{c}\right)_{\max }^{0.6} \rho_{\mathrm{mat}} K_{\rho H}\left(\frac{\mathrm{AR}_{\mathrm{HTP}}}{\cos \left(\Lambda_{0.25 \mathrm{HTP}}\right)}\right)^{0.6} \lambda^{0.04} V_{\mathrm{eH}}^{0.3}\left(\frac{c_{\mathrm{contr}}}{c}\right)^{0.4}
\end{aligned}
$$

where $\rho_{\text {mat }}$ is the density of the construction materials and $K_{\rho H}$ is the horizontal tail density factor. Both of them are the constants which must be adjusted for obtaining accurate results on actual horizontal tails. These parameters have been adjusted in such a way that applying the formula to the horizontal tail of reference aircraft, the result is accurate. The material density of this horizontal tail has been considered $1900 \mathrm{~kg} / \mathrm{m}^{3}$ and, consequently, the horizontal tail density factor was 0.0218 .

The weight of the vertical tail is a function of the vertical tail planform area, vertical tail maximum thickness-to-chord ratio, construction material, vertical tail volume coefficient, and other vertical tail geometries, such as aspect ratio, taper ratio, and sweep angle. The VTP weight is calculated as follows (in kilograms):

$$
\begin{aligned}
& W_{\mathrm{VTP}} \\
& =S_{\mathrm{VTP}} \mathrm{MAC}\left(\frac{t}{c}\right)_{\max } \rho_{\mathrm{mat}} K_{\rho V}\left(\frac{\mathrm{AR}_{\mathrm{VTP}}}{\cos \left(\Lambda_{0.25 \mathrm{VTP}}\right)}\right)^{0.6} \lambda^{0.04} V_{\mathrm{eV}}^{0.2}\left(\frac{c_{\text {contr }}}{c}\right)^{0.4}
\end{aligned}
$$

where $\rho_{\text {mat }}$ is the density of the construction materials and $K_{\rho H}$ is the vertical tail density factor. Again, these two parameters have been adjusted for obtaining accurate results for the vertical tail of reference aircraft. The material density has been taken the same as for the horizontal tail and the vertical tail density factor was 0.034. It is necessary to indicate that both tail density factors take the values approximately between the recommended values in the corresponding reference [19].

The weight of a V-tail configuration is determined from the equations presented before. The final weight will be the addition of the weight of projected horizontal tail and projected vertical tail. For each of these projections, corresponding Eq. (4) for horizontal projection or (5) for vertical projection, must be used. Some of the parameters are easier to be obtained, for example, the projected area or the span. It is calculated by multiplying the real area or real span by the sine or cosine of the dihedral angle depending on the projection, which is vertical or horizontal, respectively. From these parameters, it is possible to calculate others such as the mean aerodynamic chord or aspect ratio applying the corresponding definition. However, some other parameters are not so obvious to be obtained. This is the case of sweep of $1 / 4$ chord line or volume coefficient. The sweep of $1 / 4$ chord line varies with the dihedral angle. Equation

$$
\tan \Lambda_{0.25 H}=\frac{\tan \Lambda_{0.25}}{\cos \Gamma}
$$


shows this dependency between the sweep of the horizontal projection and the sweep of the surface. The dependency between the sweep of real surface and the vertical projection one is the same but substituting the cosine by a sine of the dihedral angle.

Regarding volume coefficient, it is necessary to recalculate the position of the mean aerodynamic chord on each projection. The position will be different in each projection. These two positions are necessary for obtaining the corresponding moment arm and, consequently, the volume coefficient.

\section{RESULTS}

\subsection{Aerodynamic Characteristics}

Aerodynamic characteristics are used for two purposes, as it has been explained previously, which are determining stability derivatives and aerodynamic forces and moments. The results of stability derivatives are referred to as the reference aircraft in order to develop a qualitative analysis. In addition, it is necessary to realize that the moment arm of the surface changes with the geometry (this fact has been analyzed before). Thus, for better comprehension of the comparison, the results of stability derivatives have been multiplied by the corresponding moment arm of each configuration. In this way, the effect of the moment arm is taken into account. Note that all derivatives are referred to as wing area and mean aerodynamic chord or span of the main wing, for longitudinal and lateral, respectively. So, it is not necessary to change the reference area or reference length because it is the same for all configurations. The analysis is conducted for concluding the effect of the different parameters. Note that the parameters considered for this study are root chord, span, taper ratio, and dihedral angle.

The results of static stability derivatives are presented in Figs. 4-6. The graphs show the combined effect of dihedral angle with taper ratio, span, and root chord, respectively. It is possible to see that dihedral angle has negative influence from the standpoint of longitudinal stability. It is true that small dihedral angles increase slightly longitudinal stability derivative, but only for angles lower than $10^{\circ}$. However, the higher dihedral angle, the higher lateral stability derivative. It is likely to expect this result because increasing dihedral angle implies that the surface is more similar to a vertical tail, which develops lateral forces in conventional tail configurations. Regarding the other three parameters, they have more influence into longitudinal stability than into lateral stability. Increasing taper ratio, root chord, or span implies higher stability derivatives. This effect is caused by the increasing of surface area and mean aerodynamic chord, which are two parameters that affect the stability derivatives. However, it is possible to see that the influence depends on the dihedral angle. On one 

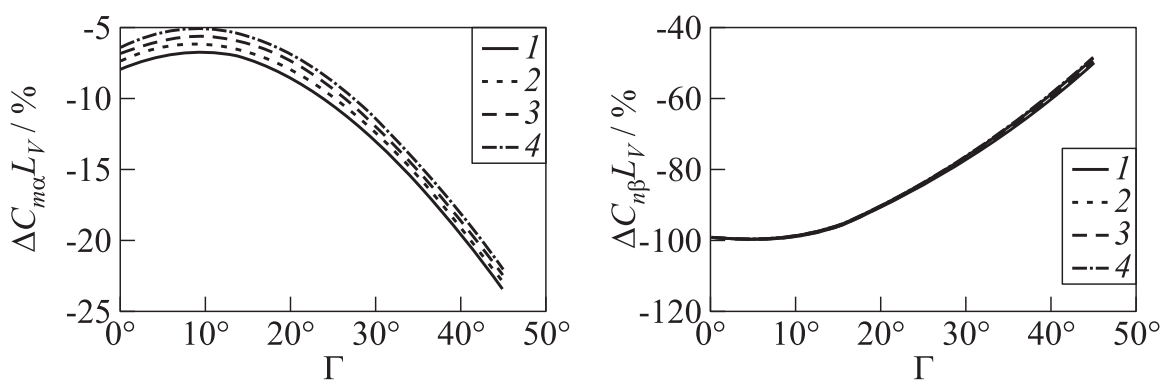

Figure 4 Variation of longitudinal and lateral static stability derivatives with dihedral angle and taper ratio: $1-0.28 ; 2-0.30 ; 3-0.32$; and $4-0.34$. The span takes the value $12 \mathrm{~m}$ and the root chord $3.9 \mathrm{~m}$. The variation is referred to the reference aircraft
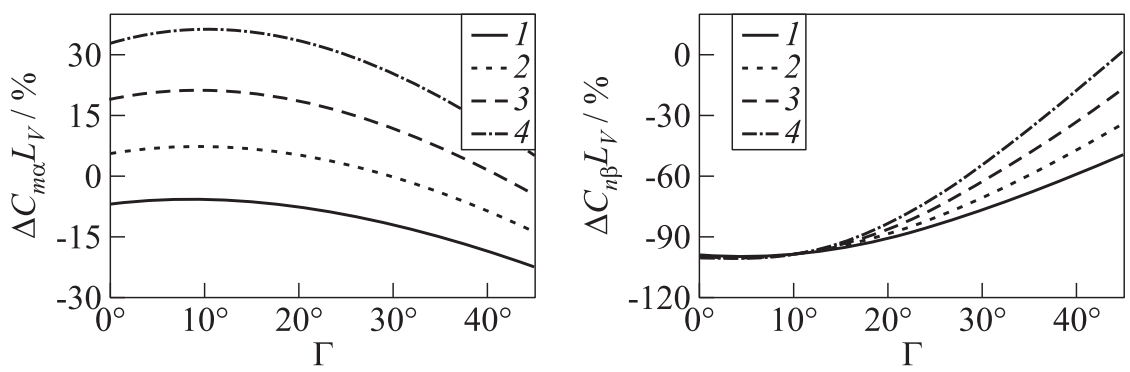

Figure 5 Variation of longitudinal and lateral static stability derivatives with dihedral angle and span: $1-12 \mathrm{~m} ; 2-14 ; 3-16$; and $4-18 \mathrm{~m}$. The taper ratio takes the value 0.32 and the root chord $3.9 \mathrm{~m}$. The variation is referred to the reference aircraft
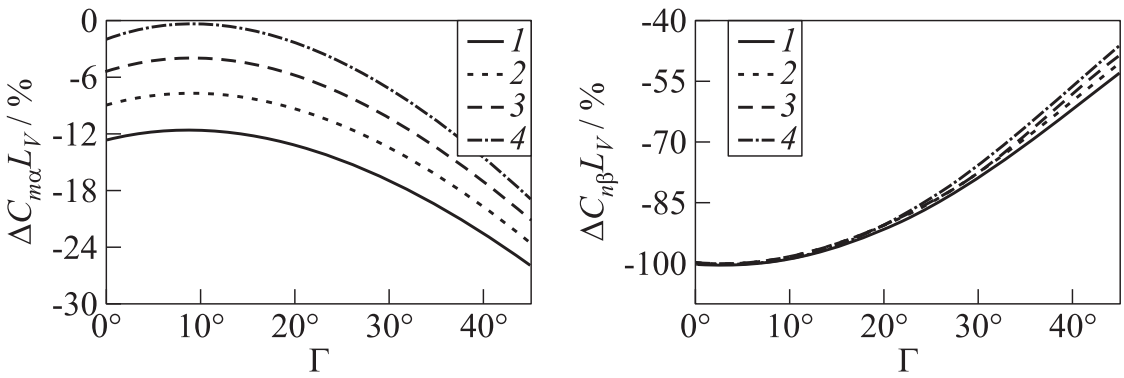

Figure 6 Variation of longitudinal and lateral static stability derivatives with dihedral angle and root chord: $1-3.3 \mathrm{~m} ; 2-3.7 ; 3-4.1$; and $4-4.5 \mathrm{~m}$. The taper ratio takes the value 0.32 and the span $12 \mathrm{~m}$. The variation is referred to the reference aircraft 
hand, the effect on longitudinal derivative is lower for high dihedral angles and, on the other hand, the effect on lateral derivative is more noticeable for high dihedral angles.

\subsection{Weight}

This subsection presents the results of applying the two methods used to estimate the weight of a V-tail configuration. The first one is based on the Farrar method and the second one is a combination of statistical formulas, as it has been described previously. The analysis has been conducted in the same way as the aerodynamic one. The effect of the geometrical parameters is presented in Figs. 7-9. Each figure shows the results of both methods, Figs. $7 a-9 a$ cor-

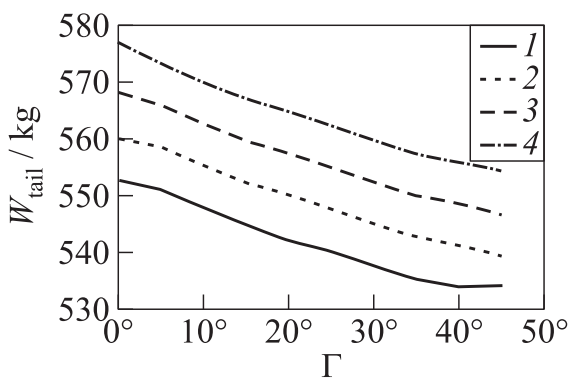

(a)

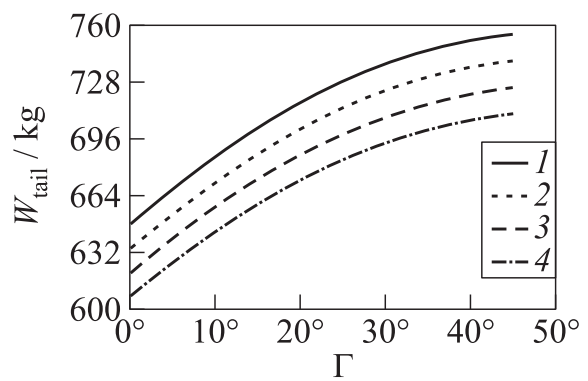

(b)

Figure 7 Variation of weight with dihedral angle and taper ratio: $1-0.28 ; 2-$ $0.30 ; 3-0.32$; and $4-0.34$. The span takes the value $12 \mathrm{~m}$ and the root chord $3.9 \mathrm{~m}$ : (a) Farrar method, and (b) statistical method

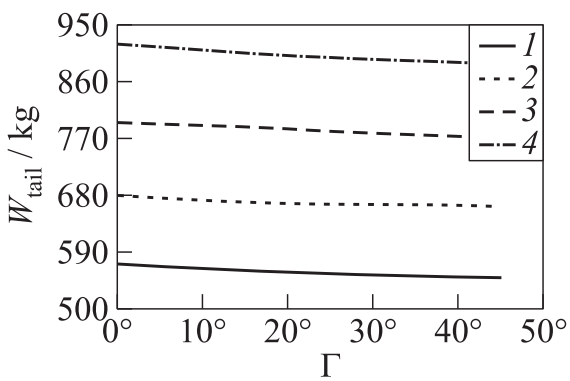

(a)

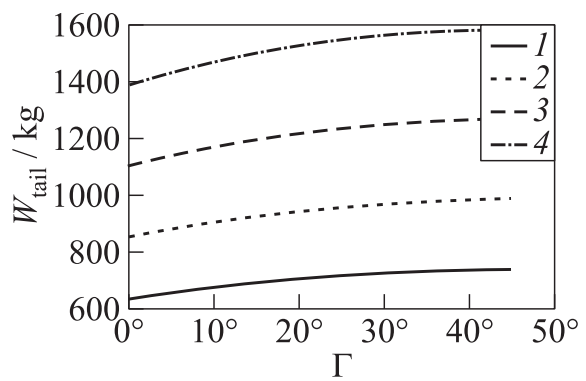

(b)

Figure 8 Variation of weight with dihedral angle and span: $1-12 \mathrm{~m} ; 2-14 ; 3-$ 16 ; and $4-18 \mathrm{~m}$. The taper ratio takes the value 0.32 and the root chord $3.9 \mathrm{~m}$ : (a) Farrar method; and (b) statistical method 


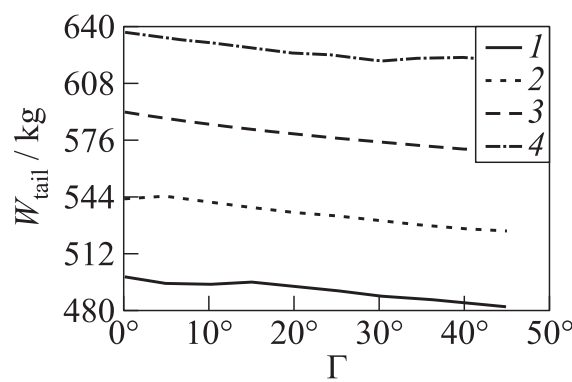

(a)

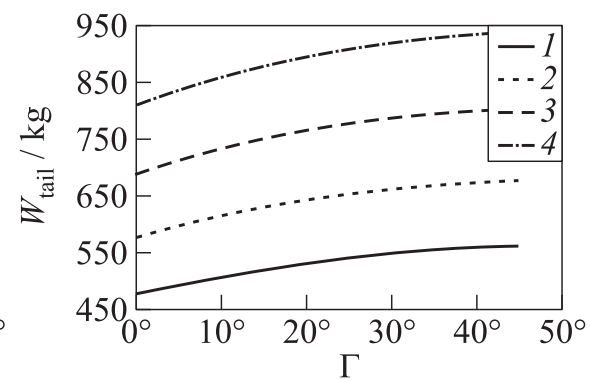

(b)

Figure 9 Variation of weight with dihedral angle and root chord: $1-3.3 \mathrm{~m} ; 2-$ $3.7 ; 3-4.1$; and $4-4.5 \mathrm{~m}$. The taper ratio takes the value 0.32 and the span $12 \mathrm{~m}$ : (a) Farrar method; and (b) statistical method

respond to Farrar method and Figs. $7 b-9 b$ to statistical method. It is possible to see that the behavior of the two methods is completely different from the standpoint of dihedral angle. The Farrar method predicts that increasing the dihedral angle supposes a reduction of the weight. On the other hand, the statistical method says exactly the opposite. The explanation is that Farrar method is incomplete, since it just considers one load case; but for high dihedral angles, probably, there is another load case that is more critical and, consequently, the behavior will change. In spite of that, this weight reduction is coherent with the hypothesis, since for high dihedral angles, the longitudinal case studies in this paper should be less critical and the final weight should be lower. This is exactly what happens in Fig. 7. The conclusion that the load case studied in this paper is not the critical one is also highlighted when comparing the two methods. It seems that the Farrar method underestimates the final weight for no dihedral angle, which should coincide with the reference horizontal tail weight. However, the statistical method predicts, with higher accuracy, the weight for no dihedral angle. Despite the differences between the two methods, both of them present similar behavior from the standpoint of the other geometrical parameters. Higher values of any parameter supposes higher weight.

\subsection{Optimization}

The optimization process has been developed with the help of MATLAB's functions. More precisely, there is a function which obtains a minimum of an objective function taking into account both linear and nonlinear constraints. This function is fmincon. For the present study, the objective function is the weight. Because two methods of estimating the weight have been presented, the same procedure 
Table 5 Bounds of parameters for optimization process

\begin{tabular}{lcccc}
\hline Boundary & Span, $\mathrm{m}$ & Dihedral angle & Taper ratio & Root chord, $\mathrm{m}$ \\
\hline Lower & 12 & $0^{\circ}$ & 0.28 & 3.3 \\
Upper & 20 & $50^{\circ}$ & 0.34 & 4.5 \\
\hline
\end{tabular}

has been repeated but changing the objective function. In this case, the constraints are the lateral and longitudinal stability derivatives, which should be at least equal to the reference aircraft ones. Note that the stability derivatives should be compared once they are multiplied by the moment arm. Furthermore, it is necessary to indicate the initial condition and the boundaries of the parameters. The parameters which are involved in this optimization process are span, dihedral angle, taper ratio, and root chord. The boundaries of each parameter are showed in Table 5 . The solution has to be maintained between these boundaries on each iteration along the calculation process. For obtaining a faster solution, it has been decided to calculate aerodynamic forces, weight, and stability derivatives for a wide combination of the parameters between the boundaries. The objective function and the constraints are determined by interpolating between the results obtained previously. This procedure allows the optimization process to be faster. The interpolation is developed with a cubic method, which is based on a cubic convolution. This method needs more computational time than a linear method, but it presents a continuity of $\mathrm{C}^{1}$ class, instead of $\mathrm{C}^{0}$ of the linear method.

Making use of some MATLAB's functions, the optimum is obtained by a sequence of different algorithms, considering each solution as the initial condition of the next algorithm. The first recommended algorithm is interior-point. It is a large-scale algorithm; so, it uses linear algebra to solve the problem that needs neither to store nor to operate on full matrices. This algorithm reaches solutions which can be slightly less accurate than those from other algorithms. The reason for this potential inaccuracy is that the (internally calculated) barrier function keeps iterates away from inequality constraint boundaries. Because of that, it is recommended to use other algorithms after it. It has been used the algorithms sqp and active-set, last. These two are the medium-scale algorithms; so, they internally create full matrices and use dense linear algebra. This reduces the inaccuracy but it requires more memory.

The results reached by the MATLAB's function are going to be verified through a feedback of the process. Because the results are obtained interpolating between matrices, it is necessary to carry out this checking. The optimal geometry will be introduced into Tornado and then, the two methods used for estimating the weight will be used. Here, a verification of the constraints will be done. At this moment, two possibilities may happen: the constraints are 
Table 6 Results of optimization process using two methods of weight estimation

\begin{tabular}{lccccc}
\hline Method & $\begin{array}{c}\text { Span, } \\
\mathrm{m}\end{array}$ & Dihedral angle & Taper ratio & $\begin{array}{c}\text { Root chord, } \\
\mathrm{m}\end{array}$ & $\begin{array}{c}\text { Weight, } \\
\mathrm{kg}\end{array}$ \\
\hline Farrar & 18.8 & $45.2^{\circ}$ & 0.28 & 3.4 & 826 \\
Statistical & 19.1 & $45.1^{\circ}$ & 0.28 & 3.3 & 1280 \\
\hline
\end{tabular}

achieved or not. In both cases, slightly variations of the parameters for the optimal geometry reached by the procedure need to be done. These variations will indicate if there were another geometries that achieve the constraints, but it was lighter. This fact could happen because the optima is obtained through interpolation, not through the whole procedure in each step, as it was mentioned before.

The geometry reached by the optimization process depends on the weight function employed. The optimal configurations are indicated in Table 6. It is possible to see that the geometry is very similar for both methods, but the weight estimation is clearly different. This fact was expectable according to the analysis developed in the previous section.

When carrying out the feedback process, it is possible to check that the constraints are achieved. Nevertheless, there is a margin in order to reach them. This margin may be caused by the interpolation method, which does not reproduce exactly the Tornado results for the stability derivatives. Hence, it is decided to vary slightly the solution and calculate the stability derivatives directly with Tornado instead of interpolating. The objective is to find another geometry that continues achieving the constraints with lower weight. This analysis shows the option of reducing the dihedral angle to $41.5^{\circ}$ and, in spite of that, the constraints continue being reached. The other parameters were maintained at the optimization process solution. Thus, the final optima is indicated in Table 7. Furthermore, Table 7 shows a comparison of stability derivatives, weight (using the statistical method), and wetted area with the reference aircraft. It is possible to see that the optima presents better longitudinal stability derivative and lower wetted area but, on the other hand, it is heavier. The reductions in wetted area suppose a benefit from the standpoint of aerodynamic drag. Finally,

Table 7 Final optima and comparison with reference tail of static stability derivatives, weight, and wetted area using statistical method

\begin{tabular}{cccccccc}
\hline $\begin{array}{c}\text { Span, } \\
\mathrm{m}\end{array}$ & $\begin{array}{c}\text { Dihedral } \\
\text { angle }\end{array}$ & $\begin{array}{c}\text { Taper } \\
\text { ratio }\end{array}$ & $\begin{array}{c}\text { Root } \\
\text { chord, } \mathrm{m}\end{array}$ & $\Delta\left(C_{m \alpha} L_{V}\right)$ & $\Delta\left(C_{n \beta} L_{V}\right)$ & $\Delta W$ & $\Delta S_{\text {wet }}$ \\
\hline 19.1 & $41.5^{\circ}$ & 0.28 & 3.3 & $+6.4 \%$ & $+0.6 \%$ & $+6 \%$ & $-34 \%$ \\
\hline
\end{tabular}



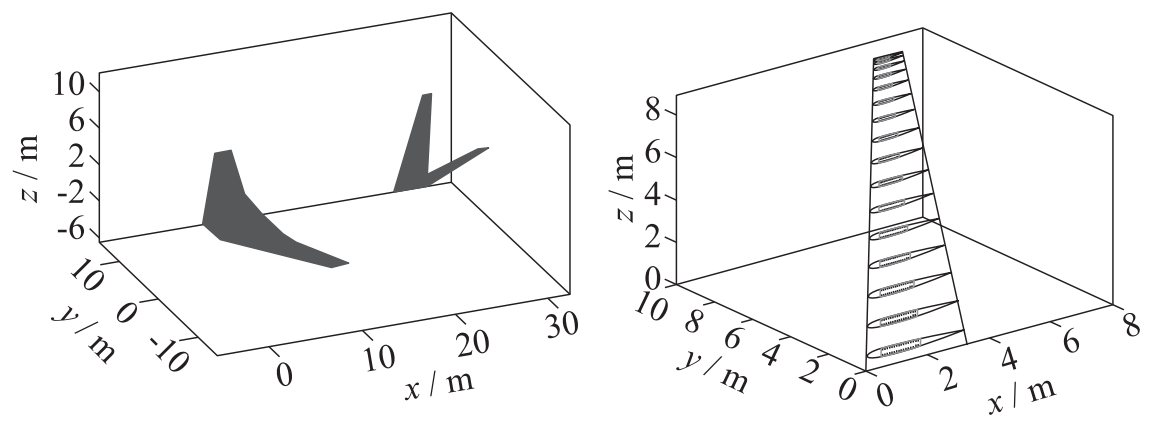

Figure 10 Three-dimensional representation of the final optima configuration selected

a three-dimensional representation of the final optima geometry is included in Fig. 10. This figure also shows the structural design reached with the Farrar method, despite the weight estimated with this method is not accurate.

\section{CONCLUDING REMARKS AND FUTURE WORKS}

This work presents a methodology of sizing an unconventional tail configuration applied to a V-tail. The sizing is carried out trying to minimize the weight. The basic geometrical parameters are taken into account in the procedure. Regarding the obtained configuration, it shows a penalization in weight with respect to the reference aircraft tail, but benefits in wetted area. Thus, it is possible to say that this study is a good first approximation to the problem.

However, this methodology should be improved in many aspects. Firstly, the Farrar method for estimating the structural weight is applicable when the structure is submitted to compression forces. It is possible that this state is not the critical one, because the tails lift both up and down in order to equilibrate the aircraft. So, it is possible that the structure is subject to a combination of tension and fatigue. It should be analyzed in order to determine which of them is more critical or simply if this state supposes an additional weight to the structure. The final objective of future work should be to develop an analogous method to this one but valid for composite materials. In this way, it will not be necessary to size the section according to metallic alloys and then supposing that the real structure presents the same geometry but using composite materials. Once this method has been developed, it could be used to validate the statistical method of estimating the weight presented in this paper.

Regarding the aerodynamic aspects, one of the main limitations of Tornado software is that it does not simulate transonic conditions. Of course, these conditions must be studied because it is probably that a transonic load state is 
the most critical. Hence, it is necessary to estimate these conditions through other procedures. It would be desirable a procedure which does not spend much computational time, in order to facilitate the work of the optimization process. Ultimately, computational fluid dynamics software will be used to these calculations. Another aspect that should be improved in this study is to increase the load cases simulated. Both lateral and longitudinal cases must be considered. These cases should be, at least, the same cases that are usually studied for the conventional tails. This fact is related to the transonic problem, because some of the load cases have transonic speeds. So, for future work, considering other more powerful software is a key recommendation.

Finally, analyzing the selection of the parameters used for this research, the work has been developed with a fixed center of gravity position. An interesting future work could be an analysis of the effect of varying its position. This study has not taken into account the effect of changing the position of the tail, it has been considered that the apex point is the same as the reference horizontal tail. For future works, this effect should be analyzed.

\section{ACKNOWLEDGMENTS}

This work has been supported by Airbus Operations Rear-End RADAR project and the Spanish Centre for Industrial Technological Development (CDTI Ministry of Economy and Competitiveness) and Airbus Operations co-funded project CURvED.

\section{REFERENCES}

1. AIRBUS. 2013. Global market forecast. Future journeys 2013-2032. Blagnac, France.

2. Boeing Commercial Airplanes. 2013. Current market outlook 2013-2032. Seattle, WA.

3. Lee, D. S., G. Pitari, V. Grewe, K. Gierens, J. E. Penner, A. Petzold, M. J. Prather, U. Schumann, A. Bais, and T. Berntsen. 2010. Transport impacts on atmosphere and climate: Aviation. Atmos. Environ. 44(37):4678-4734.

4. ICAO. 2013. Report of the Executive Committee on Agenda Item 17 (Section on Climate Change).

5. Martinez-Val, R., and E. Perez. 2009. Aeronautics and astronautics: Recent progress and future trends. Proc. Inst. Mech. Eng. C J. Mech. Eng. Sci. 223(12):2767-2820.

6. Ky, P., and B. Miaillier. 2006. SESAR: Towards the new generation of air traffic management systems in Europe. J. Air Traffic Control 48(1):11-14. 
7. Anderson, J. D., Jr. 2002. The airplane, a history of its technology. Reston, VA: AIAA. 377 p.

8. Mcmasters, J.H., and I. M. Kroo. 1998. Advanced configurations for very large transport airplanes. Aircr. Des. 1:217-242.

9. De Lucas, S., A. Velazquez, J. M. Vega, and A. Methodology. 2011. An optimization method for an aircraft rear-end conceptual design based on surrogate models. World Congress of Engineering Proceedings. III(3).

10. Kroo, I. 2005. Nonplanar wing concepts for increased aircraft efficiency. VKI lecture ser. of innovative configurations and advanced concepts for future civil aircraft. 129.

11. Ning, A., and I. Kroo. 2008. Tip extensions, winglets, and c-wings: Conceptual design and optimization. AIAA Paper No. 2008-7052. 30 p.

12. Risse, K. 2014. Design of CeRAS CSR-01. ILR RWTH Aachen University. Technical Report.

13. Melin, T. 2000. A vortex lattice MATLAB implementation for linear aerodynamic wing applications. Royal Institute of Technology (KTH). Master Thesis.

14. Lomax, T.L. 1996. Structural loads analysis for commercial transport aircraft: Theory and practice. American history through literature ser. Reston, VA: AIAA. $296 \mathrm{p}$.

15. Purser, P.E., and J. P. Campbell. 1945. Experimental verification of a simplified vee-tail theory and analysis of available data on complete models with vee-tails. NACA Report No. 823.

16. Torenbeek, E. 2013. Advanced aircraft design: Conceptual design, analysis and optimization of subsonic civil airplanes. John Wiley \& Sons, Ltd. 434 p.

17. Khun, P., J. P. Peterson, and L. R. Levin. 1952. A summary of diagonal tension. Part I: Methods of analysis. NACA Technical Note 2661.

18. Farrar, D. J. 1949. The design of compression structures for minimum weight. J. R. Aeronaut. Soc. 53(467):1041-1052.

19. Sadraey, M. H. 2013. Aircraft design: A systems engineering approach. 1st ed. John Wiley \& Sons, Ltd. 808 p. 